\title{
Letter to the Editor - Counterpoint
}

Joris R. Delanghe*, Marijn M. Speeckaert and Marc L. De Buyzere

\section{ACE Ins/Del genetic polymorphism and epidemiological findings in COVID-19}

https://doi.org/10.1515/cclm-2020-0605

Received for publication April 28, 2020; previously published online May 9,2020

Keywords: angiotensin-converting enzyme; COVID-19; polymorphism.

To the Editor,

We agree with Dr. Sadaat [1] that prevalence and mortality of COVID-19 may be attributed to a lot of variables. We have therefore chosen to construct a database of a large single continuous geographical zone to neutralize, as much as possible, a number of confounders. The determination coefficient allows calculating the percentage of the variation that can be explained by the variables included in the model.

It is clear that the majority of the observed variance in COVID-19 prevalence and mortality cannot be explained by the ACE D/I polymorphism alone. This is also the reason why we postulated that the variability in this genotype distribution might partly explain the variable prevalence of the COVID-19. The large differences in population density, testing capacity, the accurate registration of the infection fatality rate, changes in case definition [2], health policy, demographic age distribution, co-morbidities, the variation in the onset of the pandemic, ... definitely have contributed to an important variation in the pandemic statistics. So, numerous examples can be given to illustrate this variation. The presence of all these variables makes the observed association between ACE D/I polymorphism and COVID-19 [3] only stronger.

\footnotetext{
*Corresponding author: Prof. Dr. Joris R. Delanghe, Department of Clinical Chemistry, Ghent University, C. Heymanslaan 10, Gent 9000, Belgium, E-mail: joris.delanghe@ugent.be. https://orcid.org/0000-0002-5702-6792

Marijn M. Speeckaert and Marc L. De Buyzere: Department of Internal Medicine, Ghent University, Ghent, Belgium
}

Dr. Sadaat is now going much further in trying to expand the observations in the 33-countries model (with European, North-African and Middle East countries) to other continents by using a linear extrapolation of the equation derived in our study. In particular, some Far East countries (with a very different genetic pattern from the countries included in our database) $[4,5]$ have been chosen in a very selective way to disprove the correctness of the obtained equation. Taiwan and Japan are islands, which can be geographically well protected against pandemics. South Korea's only land border is a military demarcation line which does not facilitate international transport. The data of the severely struck Hubei province (high prevalence) have been diluted to the population of mainland China. Similarly, the data of the severely struck Korean Shinchenji Church have been diluted in South Korean statistics. These facts make the comparison with the European/North African/Middle East situation impossible. It is clear that the presented approach based on genetic polymorphisms has limitations: for example, the difference in COVID-19 statistics between North and South Korea is unlikely to be attributed to genetic variability. To evaluate the value of a polymorphism in a multivariate analysis, we have to select a well-defined geographical space, in which the allele frequency varies sufficiently and the classic mortality and contamination risk co-variants vary little. If one selects the whole world and include countries and peoples with large differences in BMI, cardiovascular risk, diabetes prevalence, socio-economic status, organization of health care, public health policy, ... every single polymorphism will lose its significance in a multivariate analysis evaluating the influence on infection risk and mortality due to COVID-19.

Research funding: None declared.

Author contributions: All authors have accepted responsibility for the entire content of this manuscript and approved its submission.

Competing interests: Authors state no conflict of interest. 


\section{References}

1. Saadat M. No significant correlation between ACE Ins/Del genetic polymorphism and COVID-19 infection. Clin Chem Lab Med 2020;58:1127-8.

2. Tsang TK, Wu P, Lin Y, Lau EH, Leung GM, Cowling BJ. Effect of changing case definitions for COVID-19 on the epidemic curve and transmission parameters in mainland China: a modelling study. Lancet Public Health 2020, doi: https://doi.org/10.1016/S2468-2667(20)30089-X. [Epub ahead of print].

3. Delanghe JR, Speeckaert MM, De Buyzere ML. COVID-19 infections are also affected by human ACE1 D/I polymorphism. Clin Chem Lab Med 2020;58:1125-6.

4. Reich D. Who we are and how we got here. Oxford: Oxford University Press, 2018, ISBN: 978-0-19-882125-0.

5. Cavalli-Sforza LL, Menozzi P, Piazza A. The history and geography of human genes. Princeton, NJ, USA: Princeton University Press, 1994:1-1088. 